ISSN 1997-5902

\title{
Evaluation de la qualité technologique et organoleptique de la viande de bovins de races Borgou, Lagunaire et Zébu Peulh, élevés sur des pâturages naturels
}

Salifou C.F.A. ${ }^{1}$, Dahouda M. ${ }^{2}$, Boko K.C. ${ }^{1}$, Kassa S.K. ${ }^{1}$, Houaga I. ${ }^{1}$, Farougou S. ${ }^{1}$, Mensah G.A. ${ }^{3}$, Salifou S. ${ }^{1}$, Toléba S.S. ${ }^{2}$, Clinquart A. ${ }^{4}$, Youssao A.K.I. ${ }^{*}$

1 Ecole Polytechnique d'Abomey-Calavi, Département de Production et Santé Animales, 01 BP 2009 Recette Principale, Cotonou 01, République du Bénin

${ }^{2}$ Faculté des Sciences Agronomiques, Département de Production Animale, 01 BP 526, Cotonou 01, République du Bénin

${ }^{3}$ Centre de Recherches Agricoles d'Agonkanmey, Institut National des Recherches Agricoles du Bénin ; 01 BP 884

Recette Principale, Cotonou 01, République du Bénin

${ }^{4}$ Université de Liège, Faculté de Médecine Vétérinaire, Département des Sciences des Denrées Alimentaires ; Sart

Tilman, 4000 Liège, Belgique

* Auteur de Correspondance

Prof. Dr Issaka YOUSSAO ABDOU KARIM, UAC/EPAC/Département de production et santé animales ; Tél. : (00 229) 95285988 ou (00 229) $979120 \quad 74$, Fax: (00 229) 213601 99, E-mail: iyoussao@yahoo.fr, issaka.youssao@epac.uac.bj 01 BP 2009 Cotonou 01, République du Bénin.

Original submitted in on 28th January 2013. Published online at www.m.elewa.org on 30th March 2013.

\section{RÉSUMÉ}

Objectif : La qualité de la viande prend en compte la qualité technologique, la qualité organoleptique, la qualité nutritionnelle et la qualité hygiénique. Le but de cette étude est d'évaluer les qualités technologiques et organoleptiques de la viande de bovins de races Borgou, Lagunaire et Zébu Peulh, élevés sur pâturage naturels.

Méthodologie et résultat : Des données ont été collectées de février à mars 2012 sur 10 Lagunaire, 10 Borgou et 10 Zébu Peulh. Les Zébu Peulh ont une température de refroidissement supérieure aux Borgou et les Lagunaire ont les températures les plus faibles tout au long de la maturation. La viande de bovin de race Borgou a été plus claire $(P<0,001)$ que celle des Lagunaire et des Zébu Peulh pendant que la viande des Lagunaire a une luminosité identique à celle des Zébu. Au jour d'abattage, l'indice du rouge le plus faible a été obtenu chez les zébus $(P<0,001)$. A deux jours post mortem, les Borgou ont l'indice du rouge le plus élevé $(\mathrm{P}<0,001)$. Au 8 e jour post mortem, les Lagunaires ont un indice du rouge plus élevé que les Zébu alors que l'indice du rouge de la race Borgou ne diffère pas de ceux des deux autres races. Les zébus ont les plus faibles indice du jaune alors que les Borgou ont les indices du jaune les plus élevés quel que soit le jour de mesure $(P<0,01)$. Le pH à l'abattage n'a pas varié significativement d'une race à l'autre $(P>0,05)$. La capacité de rétention d'eau de la viande de Lagunaire a été plus élevée que celles des autres races $(P<0,05)$. La viande 
des Lagunaire a été plus tendre que celle des Borgou et des Zébu. La note d'acceptation de la viande de la Lagunaire par le consommateur a été plus élevée que celles des Borgou et des Zébu $(P<0,001)$.

Conclusion et application: La viande de bovin Lagunaire a une qualité technologique, organoleptique et sensorielle meilleure à celles des Borgou et des Zébu Peulh.

Mots clés : Qualité, carcasse, viande, bovin, maturation, abattoir, Bénin

Assessment of beef technological and organoleptic qualities of Lagunaire, Borgou and Zebu Fulani bulls raised on natural pasture in Benin

\section{Abstract}

Objective: The meat quality takes into account technological quality, organoleptic quality, nutritional quality and hygienic quality. This study aims to assess beef technological and organoleptic qualities of Lagunaire, Borgou and Zebu Fulani bulls raised on natural pasture in Benin.

Methodology and results: Data were collected from February to March 2012 on 10 Lagunaire, 10 Borgou and 10 Zebu Fulani bulls. The Zebu Fulani carcass had a higher chilling temperature than the Borgou carcass and the Lagunaire carcass had the lowest chilling temperatures during maturation. Borgou breed meat was lighter $(P<0.001)$ than that of the Lagunaire and the Zebu Fulani while the meat of the Lagunaire was identical to that of the Zebu meat brightness. On the slaughter day, the lower red index was obtained in the Zebu Fulani bulls $(P<0.001)$. Two days post mortem, the Borgou had the highest red index $(P<0.001)$. On the $8^{\text {th }}$ day postmortem, the Lagunaire meat had a higher red index than the Zebu one while the Borgou breed red index didn't differ from those of the other two breeds. Zebu had the lowest index of the yellow while the Borgou had the highest index regardless of the day of measurement $(\mathrm{P}<0.01)$. The $\mathrm{pH}$ at slaughter did not vary significantly according to breed $(P>0.05)$. Water holding capacity of Lagunaire meat was higher than those of other breeds $(P<0.05)$. The beef was tenderer in Lagunaire bull than in Borgou and Zebu Fulani bulls. The consumer note of meat acceptance was higher in Lagunaire than in Borgou and Zebu Fulani bulls $(P<0.001)$.

Conclusion and application: Lagunaire has best technological and organoleptic meat qualities than those of Borgou and Zebu Fulani.

Key words: qualities, carcass, meat, maturation, slaughterhouse, Benin

\section{INTRODUCTION}

Au Bénin, la production nationale en viande est de 61646 tonnes en 2011 et la viande bovine vient en tête avec $56,75 \%$, viennent ensuite la volaille $(18,94 \%)$, les ovins et les caprins $(7,46 \%)$, les porcs $(7,46 \%)$ et enfin, les lapins et les aulacodes $(4,05 \%)$ (CountryStat, 2012). Cette production en viande est essentiellement assurée par le cheptel national bovin composé d'une diversité génétique de races dont les Lagunaire, les Somba, les Borgou et les zébus, principalement, les Zébu Peulh, White Fulani et M'Bororo Rouge. L'effectif du cheptel national bovin est estimé à 2058000 bovins en 2011 , dont $51 \%$ de race Borgou, $26 \%$ de Zébu, $5 \%$ de Lagunaire et $3 \%$ de race Somba, le reste étant constitué de métis issus de divers croisements (DE, 2011). De nombreux travaux ont été réalisés pour améliorer les performances de production et de reproduction de ces différentes races (Youssao et al., 2007 ;Youssao et al., 2009 ;Koutinhouinet al., 2009 ;Koutinhouin et al., 2010). Sur le plan sanitaire, des travaux ont été également réalisés pour améliorer la productivité des animaux (Koutinhouin et al., 2003 ;Farougou et al.; 2006; Farougou et al., 2007;DokoAllou et al., 2010 ;Zoffoun et al., 2011, Farougou et al., 2012). En aval de la production, le procédé d'abattage des bovins a été évalué en vue d'assurer la qualité sanitaire des carcasses de bovins produites aux abattoirs de Cotonou-Porto-Novo (Salifou et al., 2012a). Dans le but d'améliorer la qualité de la carcasse des bovins autochtones du Bénin, Salifou et al.(2012b) ont 
évalué les caractéristiques de la carcasse des Bovins de races Lagunaire, Borgou et Zébu Peulh élevés sur pâturages naturels. Des relations ont été également établies entre les caractéristiques de la carcasse et les composantes du $5^{\mathrm{e}}$ quartier (Salifou et al., 2012c). A l'issue de ces travaux sur la caractérisation des carcasses des races bovines du Bénin, des programmes d'amélioration pourront être établis en utilisant les différentes techniques classiques d'amélioration (systèmes d'élevage, alimentation, génétique.). Pour être plus efficace, ce programme doit innover en intégrant les aspects liés à la qualité de la viande pour rendre la filière plus compétitive. En général, le terme qualité d'un aliment regroupe : la qualité organoleptique ou sensorielle, la qualité nutritionnelle ou diététique, la qualité technologique, la qualité hygiénique (Clinquart et al., 2000 ; Cartier \& Moëvi, 2007). La présente étude se

\section{MATÉRIELS ET MÉTHODES}

Cadre de l'étude : L'abattoir de Cotonou-Porto-Novo est situé à Akpakpa sur la route Inter-Etat Bénin-Nigéria, dans le 1er Arrondissement de la Commune de Cotonou. II s'étend sur une superficie initiale de 3,5 ha $\left(6^{\circ} 21^{\prime}\right.$ de latitude Nord et $2^{\circ} 25^{\prime}$ de longitude Est). Les animaux abattus à l'abattoir de Cotonou-Porto-Novo, proviennent des Départements de l'Alibori et du Borgou pour les zébus et les Borgou. Les lagunaires y sont rarement abattus. Ceux qui ont été abattus dans le cadre de la présente étude, provenaient du Département du Zou. Ces Départements ont été déjà décrits par Salifou et al. (2012c).

Choix et abattage des animaux : Les critères de choix des animaux ont été amplement décrits par Salifou et al. (2012c). II s'agit de la race (zébu Peulh, Borgou ou Lagunaire), de l'âge ( 5 ans et déterminé à partir de la table dentaire) et du mode d'élevage (sédentaire ou transhumant, nourris au pâturage naturel et sans apport de complément alimentaire). Une fois les animaux identifiés, ils sont abattus, dépouillés, éviscérés et fendus en deux demi-carcasses. La tête, les pattes, le cuir, les viscères thoraciques, les viscères abdominaux et les deux demi-carcasses sont enfin soumis à l'inspection post mortem. Le ressuyage n'est pas pratiqué à l'abattoir de Cotonou-Porto-Novo. Au total, les Longissimus limite aux qualités technologiques et organoleptiques. Les qualités organoleptiques des viandes regroupent les propriétés sensorielles à l'origine des sensations de plaisir associées à leur consommation (Clinquart et al., 2000; Cartier \& Moëvi, 2007). La qualité sensorielle de la viande est déterminée par sa couleur, sa flaveur, sa jutosité et sa tendreté (Clinquart et al, 2000; Cartier \& Moëvi, 2007). La qualité technologique de la viande représente sa capacité à être transformée et conservée. Elle dépend du produit que l'on souhaite fabriquer (viande crue hachée et viande crue non hachée) et peut être exprimée principalement par le $\mathrm{pH}$ et par la capacité de rétention d'eau. Le but de cette étude est d'évaluer les qualités technologiques et organoleptiques de la viande de bovins de races Borgou, Lagunaire et Zébu Peulh, élevés sur des pâturages naturels.

thoracis de 30 bovins, dont 10 lagunaire, 10 Borgou et 10 Zébu Peulh ont été prélevés pour la présente étude. Pour chaque jour d'abattage, un animal par race est identifié. Collecte des données : Elle a été réalisée de février à mars 2012. Après abattage, les carcasses ont été convoyées dans un camion frigorifique vers la chambre froide de la société EREVAN en moins d'une heure. Les quatre demi-carcasses ont été placées dans la chambre froide, deux à deux sur un rail fixe et pré-identifié. La température et l'humidité relative à l'intérieure de la chambre froide ont été enregistrées. Une fois les carcasses en place, les sondes suivantes sont placées sur chaque demi-carcasse (Figure 1) :

une sonde de température en surface sous l'aponévrose superficielle du membre antérieure, en regard de l'extenseur radial du carpe, à mi-distance entre le coude et l'extrémité distale du membre antérieur ;

une sonde de pénétration en profondeur de la face interne de la cuisse à deux centimètres du fémur et en passant entre les muscles pectiné et adducteur ;

une sonde de mesure de l'humidité relative couplée à une sonde d'ambiance pendue à un crochet laissé libre entre les demi-carcasses étudiées et à une distance de $50 \pm 5 \mathrm{~cm}$ du sol. 

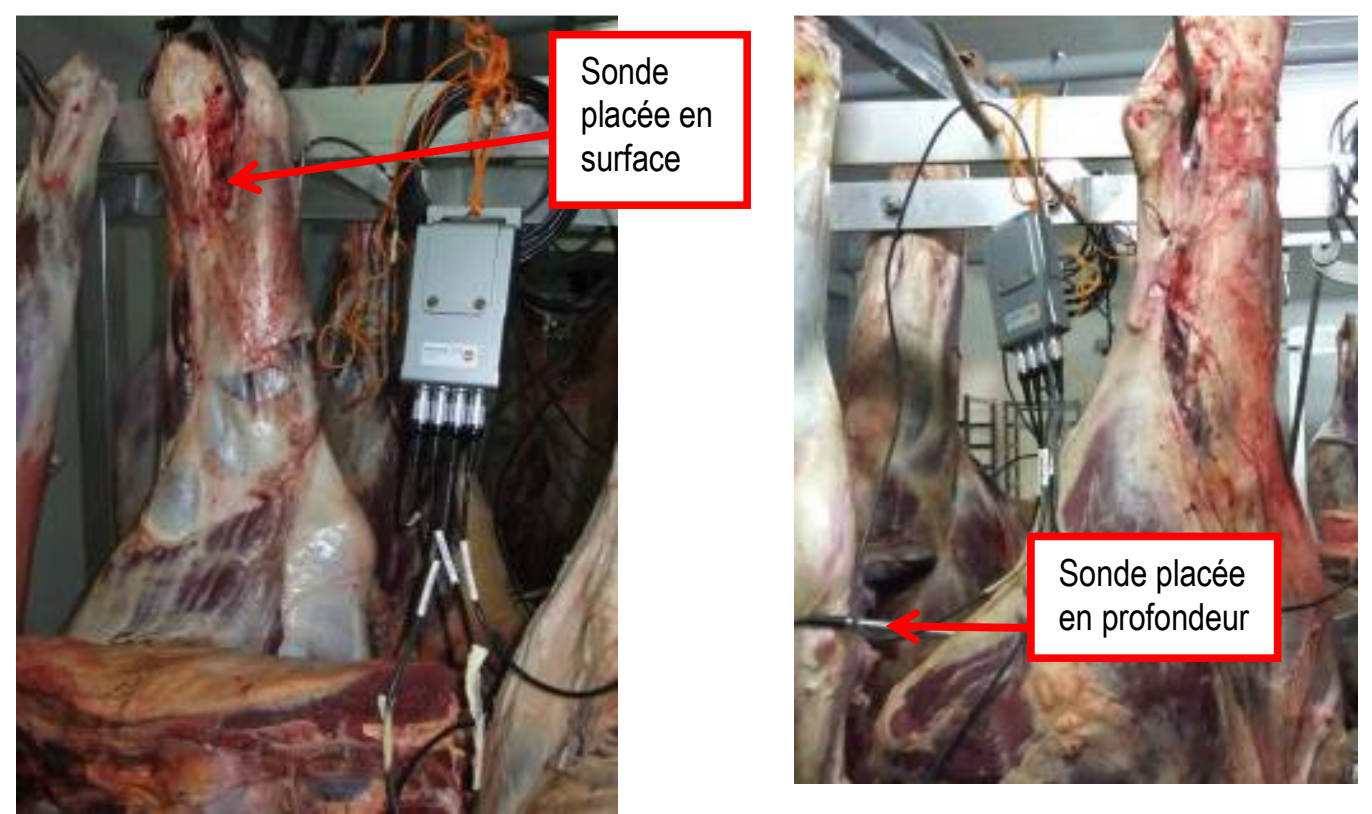

Figure 1 : Placement des dataloggersTestotermTestostor 171 , des sondes thermistances par demi- carcasse

Lors des différents tests, les demi-carcasses étaient distantes l'une de l'autre d'au $10 \mathrm{~cm}$. Les mesures ont été effectuées pendant $48 \mathrm{~h}$ dès le jour de réception des carcasses. Les mesures de la température et de l'humidité ont été enregistrées en continu à raison d'une valeur toutes les minutes pendant $48 \mathrm{~h}$. Sur chaque carcasse, le longissimus thoracis a été prélevé et divisé en 4 tranches le jour de l'abattage. La première tranche a été utilisée pour les mesures réalisées au jour d'abattage (JO), la deuxième, pour les mesures du deuxième jour post mortem (J2), la troisième, pour les mesures réalisées au jour 8 (J8) post mortem et la quatrième tranche a été utilisée pour l'analyse sensorielle. Les mesures réalisées pendant les trois périodes de la maturation étaient le $\mathrm{pH}$ et la couleur. La capacité de rétention de l'eau a été déterminée sur la tranche du jour 2. La durée de la maturation a été fixée à 8 jours. La force maximale de cisaillement a été mesurée sur l'échantillon 4 réservée à l'analyse sensorielle. Le $\mathrm{pH}$ a été mesuré 1 heure après abattage à l'aide d'un $\mathrm{pH}$ mètre portable de marque HANNA muni d'une sonde spécialisée dans le muscle longissimus thoracis au niveau des $7^{e}, 8^{e}$ et $9^{e}$ vertèbres thoraciques. Le $\mathrm{pH}$ moyen a été calculé par demi-carcasse et par carcasse. Le $\mathrm{pH}$ de chaque demi-carcasse a été la moyenne des trois mesures réalisées sur chaque demi-carcasse. Les
pH mesurés à 2 et à 8 jours post mortem ont été respectivement mesurés sur les tranches 2 et 3 . Pour chaque mesure, 5 répétitions ont été réalisées sur la tranche de longissimus thoracis. Avant toute utilisation, le $\mathrm{pH}$-mètre a été préalablement calibré avec deux étalons : $\mathrm{pH} 4$ et pH 7, suivant la procédure décrite par le fabricant (HANNA Instruments $\mathrm{R}$, Italy). La couleur a été déterminée selon les normes du Comité International d'Eclairage (CIE L* $\left.a^{*} b^{*}\right)$ après stockage des échantillons au contact de l'air atmosphérique pendant $1 \mathrm{~h} 30 \mathrm{mn}$ à $4^{\circ} \mathrm{C} . \mathrm{L}^{*}$ correspond à la luminosité, $\mathrm{a}^{*}$ l'indice du rouge et $b^{*}$ l'indice du jaune. La saturation ou chromaticité (C) et la teinte ( $h$ ) ont été déterminées respectivement suivant les formules $C=\left(a^{* 2}+b^{*}\right)^{1 / 2}$ et $h=\tan ^{-1} b^{*} / a^{*}$. Pour chaque mesure, 5 répétitions ont été réalisées sur la tranche de longissimus. La capacité de rétention d'eau (CRE) de la viande a été déterminée selon la procédure décrite par Honikel (1987). Les forces maximales de cisaillement ont été déterminées sur des carottes de viandes prélevées parallèlement au sens des fibres musculaires à l'aide d'un emporte-pièce et cuites à $75^{\circ} \mathrm{C}$ pendant une heure dans le bain marie le jour de l'analyse sensorielle. L'épreuve de cisaillement a été effectuée dans le sens perpendiculaire à l'axe des fibres musculaires à l'aide d'un textuomètre LF plus (LLYOD instruments) muni d'une lame d'épaisseur $0,42 \mathrm{~cm}$ à ouverture triangulaire 
(type Warner-Bratzler). Dix répétitions ont été faites et la force maximale de cisaillement a été exprimée en Newton (N).

L'analyse sensorielle a été faite sur chaque race (Lagunaire, Borgou et Zébu peulh) avec un panel de jury de 12 membres choisi au hasard parmi les potentiels consommateurs. Pour la dégustation, deux lots d'échantillons identiques de morceaux de $50 \mathrm{~g}$ de Longissimus thoracis ont été constitués : l'un braisé au four jusqu'à cuisson pendant 1 heure à $75^{\circ} \mathrm{C}$ et l'autre bouillit à l'eau dans une marmite sans assaisonnement et sans sel pendant également 1 heure à $75^{\circ} \mathrm{C}$. Pour chaque lot, les échantillons ont été traités séparément. Après refroidissement à la température ambiante, les échantillons de viandes cuites ont été découpés en de petits cubes identiques. Chaque juge a reçu dans une assiette de couleur différente une part de chaque découpe homologue appartenant à chaque type génétique et a rempli un formulaire de récapitulation des résultats issus de la dégustation. Par type génétique et par mode de cuisson, trois répétitions ont été faites pour chaque découpe. Les juges ont évalué les trois caractéristiques sensorielles importantes de la viande (tendreté, jutosité et la flaveur). Ces caractéristiques ont été notées sur des échelles allant de 1 à 5 . Pour la tendreté, le 1 correspond à très dur, le 2 à dure, le 3 à

\section{RÉSULTATS}

Refroidissement de la carcasse au cours de la maturation : Al'entrée en chambre froide, la température en surface est en dessous de la température en profondeur de la carcasse. L'écart entre les deux températures a été de $15,08,15,4$ et $15,10^{\circ} \mathrm{C}$, respectivement pour les races Lagunaire, Borgou et Zébu Peulh. Au fur et à mesure que le temps de conservation augmente dans la chambre froide, l'écart entre la température en profondeur et celle en surface diminuait acceptable, le 4 à tendre et le 5 à très tendre. Quant à la jutosité, le 1 correspond à très sec, le 2 à sec, le 3 à acceptable, le 4 à moelleux et le 5 à très moelleux. L'intensité de la flaveur est très faible (1), faible (2), acceptable (3), forte (4) et très forte (5). Enfin, chacun des juges a donné une note globale d'appréciation variant de 1 à 10 .

Analyses statistiques: Le logiciel Statistical Analysis System (SAS, 2006) a été utilisé pour les analyses statistiques. L'effet race a été d'abord testé pour l'analyse des données de caractéristiques technologiques de la viande. Pour chacune des races, ces caractéristiques ont été comparées en fonction du jour de maturation. Pour les caractéristiques sensorielles, les effets fixes du modèle d'analyse de variance étaient la race et le mode de cuisson. L'interaction entre race et jours de mesure a été significative et a été prise en compte dans le modèle d'analyse de variance. La procédure des modèles linéaires généralisés (SAS, 2006) a été utilisée pour l'analyse de variance. Le test de $\mathrm{F}$ a été utilisées pour déterminer la significativité de chaque effet du modèle et les moyennes moindres carrés ont été estimées et comparées deux à deux par le test de t. Les corrélations entre les différentes variables ont été déterminées par race et par la procédure Proc corr (SAS, 2006).

et devenait non significatif au-delà de $30 \mathrm{~h}$ de conservation. La température se stabilise entre 2 et $3^{\circ} \mathrm{C}$ au-delà de $40 \mathrm{~h}$ de conservation en chambre froide. De manière générale, la chute de la température est fonction de la race. Les Zébu Peulh ont une température supérieure aux Borgou. Les Lagunaire ont les températures les plus faibles tout au long de la maturation (Figure 2). 
Salifou et al.... J. Appl. Biosci. 2013. Evaluation de la qualité de la viande de bovins des races Borgou, Lagunaire et Zebu sur pâturages naturels

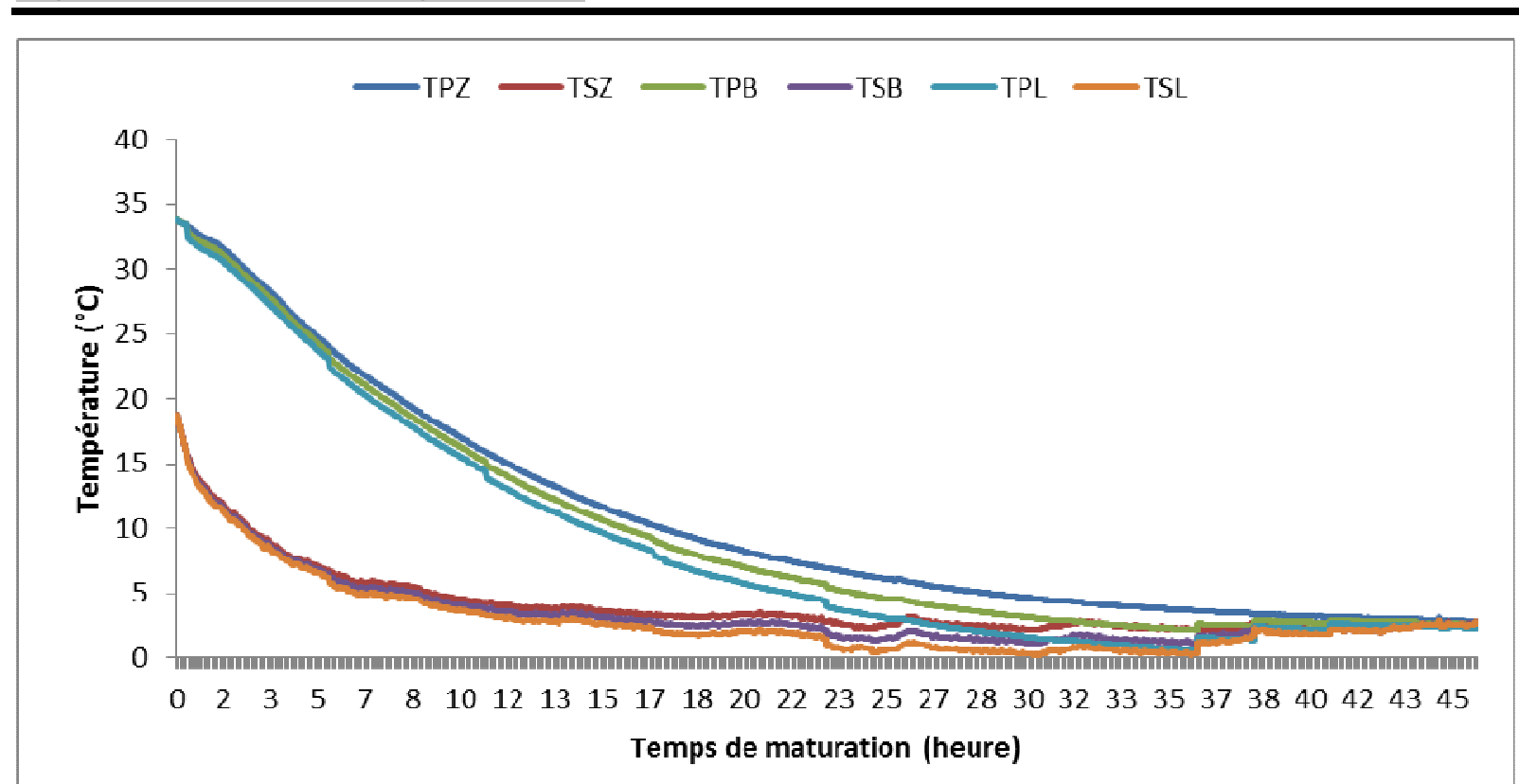

Figure 2 : Évolution de la température post mortem en chambre froide des carcasses de bovins de races Borgou, Lagunaire et Zébu Peulh

TPZ : Température en profondeur de la carcasse de zébu peulh ; PSZ : Température en surface de la carcasse de zébu peulh ; TPB : Température en profondeur de la carcasse de Borgou ; PSB : Température en surface de la carcasse de Borgou ; TPL : Température en profondeur de la carcasse de Lagunaire ; PSL : Température en surface de la carcasse de Lagunaire.

Aucune différence n'a été observée entre les $\mathrm{pH}$ des trois races pendant les $48 \mathrm{~h}$ post mortem. L'évolution du pH et de la température dans le muscle longissimus thoracis des trois races pendant les $48 \mathrm{~h}$ post mortem est présentée dans la figure 3 . Le $\mathrm{pH}$ à une heure post mortem a été de 6,82 pour une température de $31,27^{\circ} \mathrm{C}$. A 6 heures, il a été de 6,12 avec une température de $18,91^{\circ} \mathrm{C}$. De $24 \mathrm{~h}$ à $48 \mathrm{~h}$, le $\mathrm{pH}$ a varié de 5,79 à 5,72 pendant que la température variait de 4,13 à $3,92^{\circ} \mathrm{C}$.
Dans la chambre de refroidissement des carcasses, I'humidité relative a varié de 66,5 à $88,65 \%$ avec une moyenne de $76,22 \pm 3,81 \%$. Quant à la température de ladite salle, elle a été de $3,29 \pm 0,50^{\circ} \mathrm{C}$. La température la plus élevée a été $6,72^{\circ} \mathrm{C}$ et la plus faible, $1,77^{\circ} \mathrm{C}$. Des fluctuations de température et d'humidité ont été observées au cours du refroidissement (Figure 4). La corrélation entre la température et l'humidité de la salle de refroidissement a été significative $(r=0,37, P<0,001)$. 


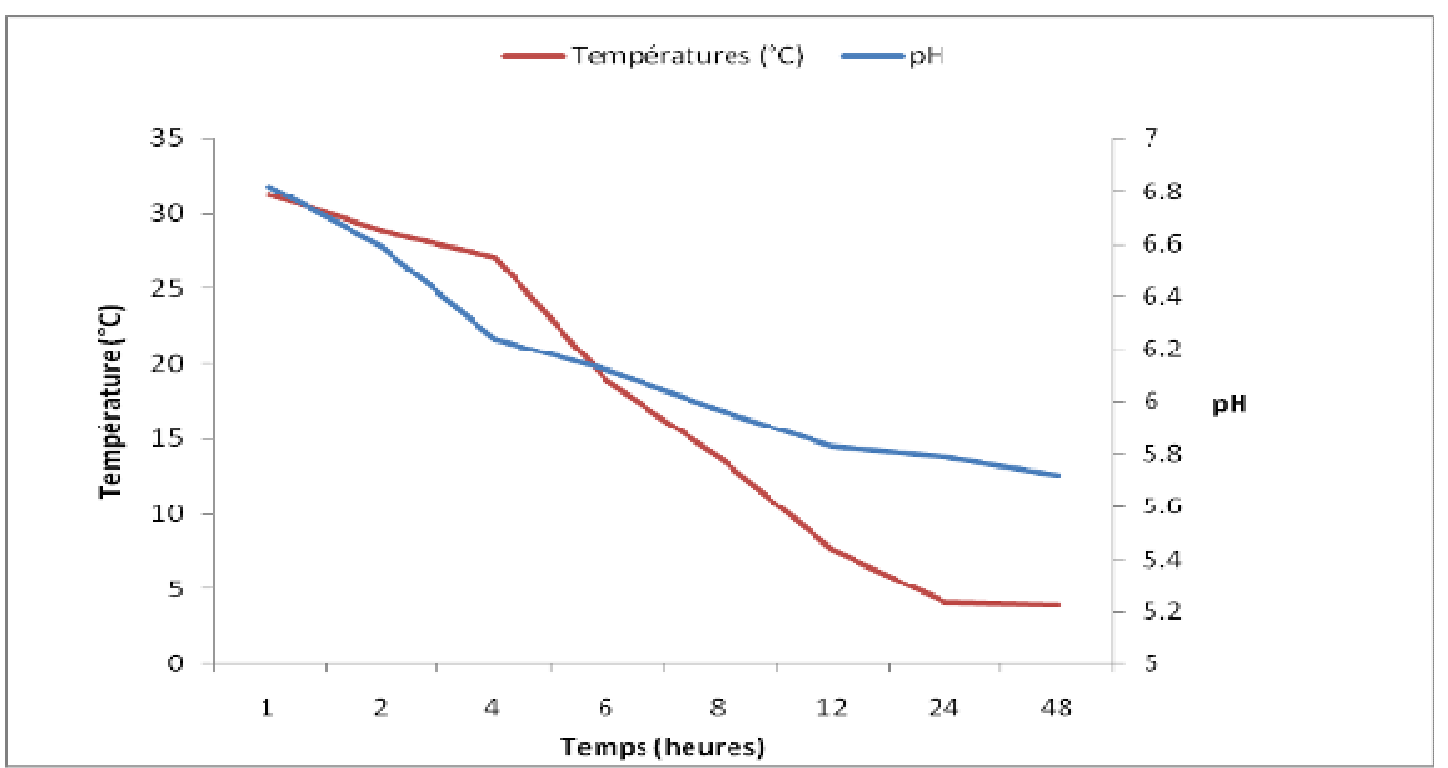

Figure 3 : Evolution du pH et de la température du muscle Longissimus thoracis pendant $48 \mathrm{~h}$ post abattage

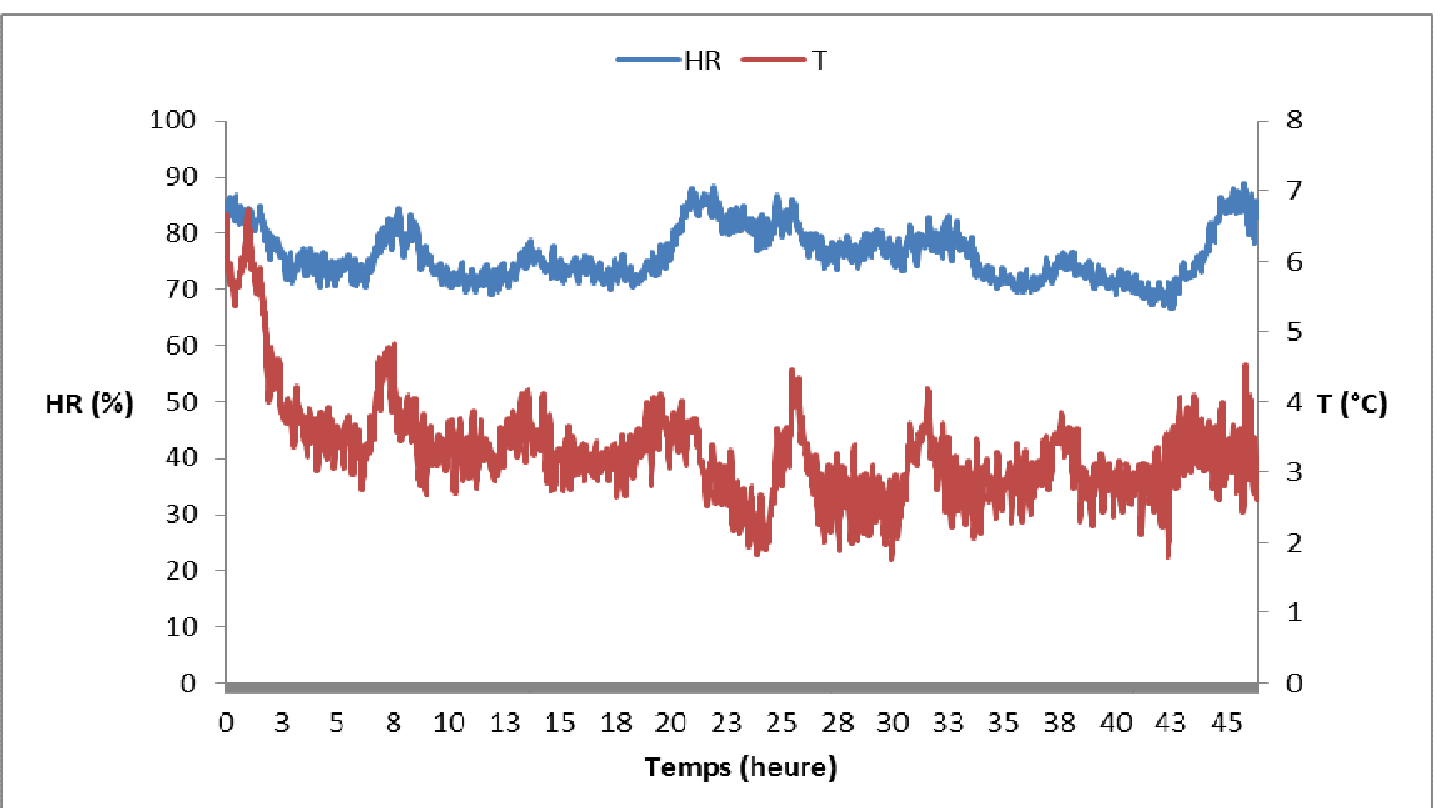

Figure 4 : Évolution de l'humidité relative (HR) et de la température dans la chambre de refroidissement (T)

Effet de la race sur les qualités technologiques de la viande : Les caractéristiques technologiques de la viande des races Lagunaire, Borgou et Zébu Peulh sont données dans le tableau 1. La viande de bovin de race Borgou a été plus claire $(P<0,001)$ que celle des Lagunaire et des
Zébu Peulh pendant que la viande des Lagunaire a une luminosité identique à celle des Zébu $(P>0,05)$ le jour d'abattage. La même tendance a été obtenue pour la luminosité des viandes des trois races aux $2^{\mathrm{e}}$ et au $8^{\mathrm{e}}$ jour post mortem. 
Tableau 1: Caractéristiques technologiques et organoleptiques de la viande de bovins de races Borgou, Lagunaire et Zébu Peulh élevés sur pâturages naturels

\begin{tabular}{|c|c|c|c|c|c|c|c|c|}
\hline \multirow[t]{2}{*}{ Variables } & \multirow[t]{2}{*}{ Jour } & \multicolumn{2}{|c|}{ Lagunaire } & \multicolumn{2}{|c|}{ Borgou } & \multicolumn{2}{|c|}{ Zébu Peulh } & \multirow[b]{2}{*}{ Test de signification } \\
\hline & & Moyenne & ES & Moyenne & ES & Moyenne & ES & \\
\hline \multirow{3}{*}{$L^{*}(\%)$} & Jo & $31,54 b$ & 0,60 & $34,42 a$ & 0,67 & $30,14 b$ & 0,67 & *** \\
\hline & $\mathrm{J} 2$ & $35,46 b$ & 0,62 & $37,07 a$ & 0,71 & $34,35 b$ & 0,71 & * \\
\hline & J8 & $33,52 b$ & 0,87 & $36,47 a$ & 0,97 & $32,36 \mathrm{~b}$ & 0,97 & * \\
\hline \multirow{3}{*}{$a^{*}$} & JO & $21,82 a$ & 0,58 & $22,61 a$ & 0,65 & $18,24 b$ & 0,65 & $* * *$ \\
\hline & J2 & $18,01 b$ & 0,69 & $23,09 a$ & 0,77 & $19,72 b$ & 0,77 & $* * *$ \\
\hline & J8 & $16,79 a$ & 0,62 & $15,65 a b$ & 0,70 & $13,76 b$ & 0,70 & $* *$ \\
\hline \multirow{3}{*}{$b^{*}$} & JO & $9,62 a$ & 0,46 & $10,33 a$ & 0,52 & $7,75 b$ & 0,52 & ** \\
\hline & J2 & $11,76 a b$ & 0,43 & $12,99 a$ & 0,49 & $11,21 b$ & 0,49 & * \\
\hline & J8 & $7,39 a b$ & 0,57 & $9,90 \mathrm{a}$ & 0,64 & $7,01 b$ & 0,64 & ** \\
\hline \multirow{3}{*}{ Teinte } & J0 & $2,20 a$ & 0,07 & $2,10 a$ & 0,07 & $2,23 a$ & 0,07 & NS \\
\hline & J2 & $1,33 b$ & 0,04 & $1,59 a$ & 0,04 & $1,58 \mathrm{a}$ & 0,04 & $\star * *$ \\
\hline & $\mathrm{J} 8$ & $2,57 a$ & 0,16 & $1,41 b$ & 0,18 & $1,91 \mathrm{ab}$ & 0,18 & $* * *$ \\
\hline \multirow{3}{*}{ Chromaticité } & J0 & $23,88 a$ & 0,7 & $24,9 a$ & 0,78 & $19,84 b$ & 0,78 & 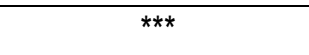 \\
\hline & $\mathrm{J} 2$ & $21,56 b$ & 0,78 & $26,5 a$ & 0,88 & $22,70 b$ & 0,88 & $* \star *$ \\
\hline & J8 & $18,62 a$ & 0,68 & $18,6 a$ & 0,76 & $15,57 \mathrm{~b}$ & 0,76 & ** \\
\hline \multirow{3}{*}{$\mathrm{pH}$} & J0 & $6,28 a$ & 0,09 & $6,50 a$ & 0,10 & $6,55 a$ & 0,10 & NS \\
\hline & $\mathrm{J} 2$ & $5,63 a$ & 0,06 & $5,76 a$ & 0,09 & $5,60 \mathrm{a}$ & 0,07 & NS \\
\hline & J8 & $5,65 a$ & 0,02 & $5,67 a$ & 0,13 & $5,61 a$ & 0,04 & NS \\
\hline PJE(\%) & J0 & $4,91 a$ & 0,65 & $3,63 b$ & 0,73 & $4,55 b$ & 0,73 & * \\
\hline $\mathrm{PJC}(\%)$ & $\mathrm{J} 2$ & $41,52 a$ & 0,77 & $38,9 \mathrm{~b}$ & 0,86 & $37,18 b$ & 0,86 & ** \\
\hline CRE $(\%)$ & J2 & $46,43 a$ & 1,19 & $42,53 b$ & 1,33 & $41,73 b$ & 1,33 & $* *$ \\
\hline Texture (N) & & $35,9 a$ & 12,4 & $62,1 a$ & 12,4 & $40,4 a$ & 12,4 & NS \\
\hline
\end{tabular}

$L^{*}$ : luminosité ; $a^{*}$ : indice du rouge ; $b^{*}$ : indice du jaune ; PJE : Perte de jus à l'écoulement ; PJC : Perte de jus à la cuisson ; CRE : Capacité de Rétention d'Eau ; J0 : jour zéro ; J2: Jour deux ; J8 : Jour huit ; ES : Erreur Standard ; ${ }^{* *}: P<0,001$; les moyennes de la même ligne suivies des lettres différentes, diffèrent significativement au seuil de $5 \%$ 
Tableau 2: Évolution des caractéristiques technologiques et organoleptiques de la viande de bovins de races Borgou, Lagunaire et Zébu Peulh au cours de la maturation

\begin{tabular}{|c|c|c|c|c|c|c|c|c|c|c|c|}
\hline \multirow[t]{2}{*}{ Variables } & \multicolumn{3}{|c|}{ Zébu Peulh } & \multicolumn{3}{|c|}{ Borgou } & \multicolumn{3}{|c|}{ Lagunaire } & \multirow[t]{2}{*}{ DSR } & \multirow[t]{2}{*}{ ANOVA } \\
\hline & Jo & $\mathrm{J} 2$ & J8 & Jo & $\mathrm{J} 2$ & J8 & Jo & $\mathrm{J} 2$ & J8 & & \\
\hline$L^{*}(\%)$ & $30,14^{a}$ & $34,35^{b}$ & $32,36^{a b}$ & $34,42^{b}$ & $37,07^{a}$ & $36,47^{a b}$ & $31,54^{b}$ & $35,46^{a}$ & $33,52^{\mathrm{ab}}$ & 0,72 & $* * *$ \\
\hline$a^{*}$ & $18,24^{a}$ & $19,72^{\mathrm{a}}$ & $13,76^{b}$ & $22,62^{a}$ & $23,09 a$ & $15,65^{b}$ & $21,82^{a}$ & $18,01^{b}$ & $16,79^{b}$ & 0,68 & $* * *$ \\
\hline$b^{*}$ & $7,76^{a}$ & $11,21^{b}$ & $7,01^{a}$ & $10,33^{b}$ & $12,99 a$ & $9,90^{b}$ & $9,62^{a}$ & $11,76^{b}$ & $7,39 c$ & 0,57 & $* * *$ \\
\hline Teinte & $2,24^{a}$ & $1,58^{b}$ & $1,91^{\mathrm{ab}}$ & $2,10^{\mathrm{a}}$ & $1,59^{b}$ & $1,41^{b}$ & $2,20^{b}$ & $1,33^{c}$ & $2,57^{\mathrm{a}}$ & 0,07 & $* * *$ \\
\hline Chromaticité & $19,84^{a}$ & $22,70^{b}$ & $15,57^{c}$ & $24,90^{a}$ & $26,50^{a}$ & $18,60^{b}$ & $23,88^{a}$ & $21,56^{b}$ & $18,62^{c}$ & 0,87 & $* * *$ \\
\hline $\mathrm{pH}$ & $6,55^{a}$ & $5,60^{\mathrm{b}}$ & $5,61^{b}$ & $6,50^{\mathrm{a}}$ & $5,76^{b}$ & $5,67^{\mathrm{b}}$ & $6,28^{a}$ & $5,63^{b}$ & $5,65^{b}$ & 0,09 & ** \\
\hline
\end{tabular}

$L$ : luminosité ; $a^{*}$ : indice du rouge ; $b^{*}$ : indice du jaune ; $F$ : Force de cisaillement ; J0 : jour zéro ; J0 : Jour deux ; J8 : Jour huit ; DSR : Déviation Standard Résiduelle; ${ }^{* * *}: P<0,001$; ANOVA : Test de significativité; les moyennes intra classes de la même ligne suivie des lettres différentes, diffèrent significativement au seuil de $5 \%$.

Tableau 3a: Caractéristiques organoleptiques de la viande de bovins de race Borgou Lagunaire et Zébu

\begin{tabular}{|c|c|c|c|c|c|c|c|c|c|c|c|c|}
\hline \multirow[t]{3}{*}{ Variables } & \multicolumn{6}{|c|}{ Race } & \multicolumn{4}{|c|}{ Cuisson } & \multicolumn{2}{|c|}{ Test de signification } \\
\hline & \multicolumn{2}{|c|}{ Borgou } & \multicolumn{2}{|c|}{ Lagunaire } & \multicolumn{2}{|c|}{ Zébu } & \multicolumn{2}{|c|}{ Bouillie } & \multicolumn{2}{|c|}{ Braisée } & \multirow[b]{2}{*}{ Race } & \multirow[b]{2}{*}{ Cuisson } \\
\hline & Moyenne & DS & Moyenne & DS & Moyenne & DS & Moyenne & DS & Moyenne & DS & & \\
\hline Flaveur & $2,30^{a}$ & 0,73 & $2,60^{a}$ & 0,88 & $2,30^{a}$ & 1,26 & $2,47^{a}$ & 0,97 & $2,33^{a}$ & 0,99 & NS & NS \\
\hline Jutosité & $2,80^{a}$ & 0,83 & $2,95^{\mathrm{a}}$ & 0,83 & $2,90^{\mathrm{a}}$ & 0,91 & $2,97^{a}$ & 0,76 & $2,80^{a}$ & 0,92 & NS & NS \\
\hline Tendreté & $2,05^{a}$ & 0,83 & $2,80^{b}$ & 0,89 & $1,90^{\mathrm{a}}$ & 1,02 & $2,37^{a}$ & 0,93 & $2,13^{a}$ & 1,04 & ** & NS \\
\hline Note & $5,47^{b}$ & 1,44 & $6,69 \mathrm{a}$ & 1,79 & $5,22^{b}$ & 1,35 & $5,63^{a}$ & 1,53 & $5,96^{a}$ & 1,76 & * & NS \\
\hline
\end{tabular}

DS : Déviation standard ; : $P<0,05 ;{ }^{*}: P<0,01 ; N S: P>0,05 ;$ les moyennes de la même ligne suivie des lettres différentes, diffèrent significativement au seuil de $5 \%$

Tableau 3b: Interaction entre race et mode de cuisson 


\begin{tabular}{|c|c|c|c|c|c|c|c|c|c|c|c|c|c|}
\hline \multirow[t]{3}{*}{ Variables } & \multicolumn{4}{|c|}{ Borgou } & \multicolumn{4}{|c|}{ Lagunaire } & \multicolumn{4}{|c|}{ Zébu } & \multirow[t]{3}{*}{ Test de signification } \\
\hline & \multicolumn{2}{|c|}{ Bouillie } & \multicolumn{2}{|c|}{ Braisée } & \multicolumn{2}{|c|}{ Bouillie } & \multirow{2}{*}{$\begin{array}{l}\text { Braisée } \\
\text { Moyenne }\end{array}$} & & \multicolumn{2}{|c|}{ Bouillie } & \multicolumn{2}{|c|}{ Braisée } & \\
\hline & Moyenne & DS & Moyenne & DS & Moyenne & DS & & DS & Moyenne & DS & Moyenne & DS & \\
\hline Flaveur & $2,30^{a}$ & 0,82 & $2,30^{a}$ & 0,67 & $2,70^{a}$ & 0,67 & $2,50^{a}$ & 1,08 & $2,40^{a}$ & 1,35 & $2,20^{a}$ & 1,23 & NS \\
\hline Jutosité & $2,90^{a}$ & 0,74 & $2,70^{a}$ & 0,95 & $2,80^{a}$ & 0,79 & $3,10^{a}$ & 0,88 & $3,20^{a}$ & 0,79 & $2,60^{a}$ & 0,97 & NS \\
\hline Tendreté & $2,10^{a}$ & 0,88 & $2,00^{a}$ & 0,82 & $2,50^{a}$ & 0,85 & $3,10^{a}$ & 0,88 & $2,50^{a}$ & 1,08 & $1,30^{\mathrm{b}}$ & 0,48 & ** \\
\hline Note & $5,39 a$ & 1,45 & $5,56^{a}$ & 1,51 & $5,94^{a}$ & 1,81 & $7,44^{a}$ & 1,51 & $5,56^{a}$ & 1,42 & $4,89^{a}$ & 1,27 & NS \\
\hline
\end{tabular}

DS : Déviation standard ; ** : P<0,01; NS : P>0,05; les moyennes de la même ligne suivie des lettres différentes, diffèrent significativement au seuil de $5 \%$ 

Lagunaire et Zebu sur pâturages naturels

Au jour d'abattage, l'indice du rouge le plus faible a été obtenu chez les zébus $(P<0,001)$ tandis qu'aucune différence n'a été observée entre les Borgou et les Lagunaire $(P>0,05)$. A deux jours post mortem, les Borgou ont l'indice du rouge le plus élevé. Au $8^{\mathrm{e}}$ jour post mortem, les Lagunaires ont un indice du rouge plus élevé que les Zébu alors qu'aucune différence significative n'a été observée entre l'indice du rouge de la race Borgou et ceux des deux autres races. Quant à l'indice du jaune, les zébus ont présenté les plus faibles indice alors que les Borgou ont les indices les plus élevés quel que soit le jour de mesure $(P<0,01)$. Les Lagunaire ont un indice du jaune identique à celui des Borgou, quel que soit le jour de mesure au cours de la maturation. La teinte n'a pas varié d'une race à l'autre le jour d'abattage. Toutefois, les teintes les plus faibles ont été respectivement obtenues chez les Lagunaire au jour $2(P<0,001)$ et chez les Borgou au jour 8 post mortem $(P<0,001)$. Les valeurs de chromaticité les plus élevées ont été enregistrées chez les Lagunaire et les Borgou à l'abattage et au $8^{\mathrm{e}}$ jour post mortem $(P<0,01)$ tandis qu'au jour 2 , la viande de la race Borgou a la valeur de la chromaticité la plus élevée $(\mathrm{P}<0,001)$. Le $\mathrm{pH}$ à l'abattage n'a pas varié significativement d'une race à l'autre pour le même jour de mesure. La perte de jus à l'égouttage, la perte de jus à la cuisson et la capacité de rétention d'eau de la viande des lagunaires ont été plus élevées que celles des autres races. La race Borgou et le Zébu Peulh ont des capacités de rétention d'eau identiques, il en est de même pour les pertes de jus à l'égouttage et à la cuisson. La force de cisaillement n'a pas significativement varié d'une race à l'autre. Toutefois, les forces de cisaillement les plus élevées ont été obtenues chez les Borgou et les plus faibles ont été enregistrées chez les Lagunaire.

Variation intra-races de la qualité technologique au cours de la maturation: Le tableau 2 présente les résultats des caractéristiques technologiques de la viande de chacune des races bovines au cours de la maturation. Pour chacune d'elles, la luminosité a significativement augmenté du jour d'abattage au jour 2 post mortem et a significativement diminué au 8 e jour post mortem $(P<0,001)$. Quant à l'indice du rouge, sa teneur a augmenté de manière non significative du jour d'abattage au jour 2 post mortem avant de chuter significativement au jour 8 post mortem chez les Borgou et les zébus. Par contre, chez les Lagunaire, l'indice du rouge a augmenté significativement du jour d'abattage au jour 2, pour chuter du jour 2 au jour 8 au cours de la maturation $(P<0,001)$. Quant à l'indice du jaune, il a augmenté de $\mathrm{J}_{0}$ à $\mathrm{J}_{2}$ et a ensuite diminué de J2 à J8 quel que soit la race. La teinte a diminué progressivement pendant toute la durée dematuration chez la race Borgou et pendant les premières $48 \mathrm{~h}$ chez les Zébus. Chez les Lagunaire, la teinte a diminué significativement pendant les premières $48 \mathrm{~h}$ avant d'augmenter pour le reste du temps de la maturation. Pendant les $48 \mathrm{~h}$ post mortem, la chromaticité a été constante chez les Borgou pendant qu'elle a augmenté chez les Lagunaire et les zébus. Quel que soit la race, les valeurs de la chromaticité ont été les plus faibles à la fin de la maturation. Le pH a chuté significativement pendant les premières $48 \mathrm{~h}$ post mortem chez les trois races et n'a plus significativement varié jusqu'au 8 jour de la maturation.

Caractéristiques organoleptiques de la viande de bovins: La flaveur et la jutosité de la viande de chacune des trois races n'ont pas varié d'une race à l'autre selon les consommateurs. Par contre, la viande des Lagunaire a été plus tendre que celle des Borgou et des Zébu $(P<0,01)$. De manière globale, en prenant en compte la flaveur, la jutosité et la tendreté, la note d'acceptation de la viande de la Lagunaire par le consommateur a été plus élevée que celles des Borgou et des Zébus $(P<0,05)$. Quant au mode de cuisson, aucune différence n'a été observée entre la viande bouillie et la viande braisée sur la tendreté, la jutosité, la flaveur et la note globale d'acception par le consommateur (tableau 3a). Chez les Borgou et les Lagunaire, aucune différence significative n'a été observée entre les caractéristiques organoleptiques de la viande (tableau $3 b$ ). Par contre, chez les zébus, la viande bouillie a été plus tendre que la viande braisée. La tendreté a été fortement corrélée à la flaveur $(r=0,583, P<0,01)$ chez la race Borgou, à la jutosité chez la Lagunaire $(r=0,485, P<0,05)$ et chez les zébus $(r=0,610, P<0,610)$. Chez chacune des trois races, la corrélation entre la force de cisaillement et la tendreté a été hautement significative $(P<0,001)$. Tout comme la tendreté, la force de cisaillement a été positivement corrélée à la flaveur chez la race Borgou, à la jutosité chez la Lagunaire et chez les Zébu. Quant à la note d'acception globale, elle a été fortement corrélée à la jutosité chez la Lagunaire et chez le zébu $(P<0,05)$ et fortement associée à la tendreté chez les Lagunaire (tableau 4). 


\section{Salifou et al.... J. Appl. Biosci. 2013. Evaluation de la qualité de la viande de bovins des races Borgou,}

Lagunaire et Zebu sur pâturages naturels

Tableau 4: Corrélations entre les caractéristiques organoleptiques de la viande des races bovines Borgou, Lagunaire et Zébu

\begin{tabular}{llcccc}
\hline Race & Variable & Jutosité & Tendreté & Note & Texture \\
\hline Borgou & Flaveur & $0,362^{\mathrm{NS}}$ & $0,583^{* *}$ & $0,142^{\mathrm{NS}}$ & $0,565^{* *}$ \\
& Jutosité & & $0,398^{\mathrm{NS}}$ & $0,339^{\mathrm{NS}}$ & $0,348^{\mathrm{NS}}$ \\
& Tendreté & & & $0,438^{\mathrm{NS}}$ & $0,913^{* * *}$ \\
& Note & & & & $0,455^{\mathrm{NS}}$ \\
\hline Lagunaire & Flaveur & $0,332^{\mathrm{NS}}$ & $0,293^{\mathrm{NS}}$ & $0,163^{\mathrm{NS}}$ & $0,398^{\mathrm{NS}}$ \\
& Jutosité & & $0,485^{*}$ & $0,496^{*}$ & $0,571^{* *}$ \\
& Tendreté & & & $0,643^{* *}$ & $0,897^{* * *}$ \\
& Note & & & & $0,498^{*}$ \\
\hline Zébu Peulh & Flaveur & $0,394^{\mathrm{NS}}$ & $0,188^{\mathrm{NS}}$ & $0,022^{\mathrm{NS}}$ & $0,291^{\mathrm{NS}}$ \\
& Jutosité & & $0,610^{* *}$ & $0,575^{*}$ & $0,585^{* *}$ \\
& Tendreté & & $0,462^{\mathrm{NS}}$ & $0,907^{* * *}$ \\
& Note & & & & $0,505^{*}$ \\
\hline
\end{tabular}

NS : $P>0,05 ;{ }^{*}: P<0,05,{ }^{* *}: P<0,01,{ }^{* * *}: P<0,001 ;$

\section{DISCUSSION}

Refroidissement de la carcasse au cours de la maturation: En dehors des supermarchés, la maturation ne se fait pas au Bénin et dans la plupart des pays de l'Afrique Subsaharienne. La viande est consommée le jour d'abattage sans être maturée. L'évolution de la température de la carcasse au cours de la maturation a été évaluée dans les conditions des supermarchés sur les races autochtones du Bénin. Ainsi, les Zébu Peulh ont une température supérieure aux Borgou et les Lagunaire ont les températures les plus faibles tout au long du refroidissement. Cette différence est sans doute liée aux poids des carcasses des races bovines abattues. Plus le poids de la carcasse est élevé, plus lent est le refroidissement. Selon Salifou et al. (2012b), les carcasses de Zébu Peulh sont plus lourdes que celles des Borgou pendant que les Lagunaires ont les carcasses les plus légères. Au fur et à mesure que le temps de conservation augmente dans la chambre froide, l'écart entre la température en profondeur et celle en surface diminuait pour atteindre 2 à $3^{\circ} \mathrm{C}$ vers $30 \mathrm{~h}$ et devenait non significatif chez chacune des trois races. Selon le Règlement (CE) No 853/2004 du Parlement européen et du Conseil du 29 avril 2004 fixant des règles spécifiques d'hygiène applicables aux denrées alimentaires d'origine animale, l'inspection post mortem doit être suivie immédiatement d'une réfrigération dans l'abattoir afin d'assurer, dans toutes les parties de la viande, une température ne dépassant pas $3^{\circ} \mathrm{C}$ pour les abats et $7{ }^{\circ} \mathrm{C}$ pour les autres viandes, selon une courbe assurant une diminution continue de la température.
Dans la présente étude, cette courbe a suivi la tendance recommandée, mais la mise en chambre froide est faite 2 à $3 \mathrm{~h}$ après l'inspection à la température ambiante (plus ou moins $30^{\circ} \mathrm{C}$ ), précurseur d'une maturation précoce. Ce retard à la mise en chambre froide s'explique par le fait que celle-ci devant servir de lieu de refroidissement est située hors de la zone d'implantation de l'abattoir et qu'il faut des procédures administratives avant que les carcasses ne soient convoyées dans un camion frigorifique. Les températures en surface $\left(20,18 \pm 2,83^{\circ} \mathrm{C}\right)$ et en profondeur $\left(35,44 \pm 3,15^{\circ} \mathrm{C}\right)$ des carcasses juste à l'entrée dans la chambre froide montraient un début de refroidissement ayant eu lieu dans le camion frigorifique. Delhalle et al. (2010) ont enregistré une température de 17 à $22^{\circ} \mathrm{C}$ environ à la surface et de 40 à $42^{\circ} \mathrm{C}$ environ en profondeur des carcasses de bovins blanc bleu belge dans différents abattoirs en Belgique, immédiatement après l'inspection post mortem.

La chute de la température aussi bien en surface qu'en profondeur de la carcasse des trois races bovines, est dans les limites tolérables pour éviter le développement microbien et le phénomène de contracture au froid défavorable à la tendreté. Cartier \& Moevi (2007) préconisent qu'il faut en $10 \mathrm{~h}$ de réfrigération descendre en-dessus de $20^{\circ} \mathrm{C}$ mais rester au-dessus de $10^{\circ} \mathrm{C}$ au sein de la carcasse. CSIRO (1989) recommande d'atteindre une température de $15^{\circ} \mathrm{C}$ en moins de $24 \mathrm{~h}$ pour éviter une prolifération microbienne probable. Dans la présente étude, au bout de $10 \mathrm{~h}$ de réfrigération, la température en profondeur des carcasses était comprise 


\section{Salifou et al.... J. Appl. Biosci. 2013. Evaluation de la qualité de la viande de bovins des races Borgou,}

Lagunaire et Zebu sur pâturages naturels

entre $21,27^{\circ} \mathrm{C}$ et $24,31^{\circ} \mathrm{C}$. Ces températures, sont en dessous des valeurs enregistrées $\left(27,5-28,5^{\circ} \mathrm{C}\right.$ à 10 heures post mortem) par Collignon (2009), ce qui élimine tout risque de multiplication microbienne à $10 \mathrm{~h}$ postmortem. Un mauvais refroidissement de la carcasse favorise la contamination de la carcasse par Listeria monocytogenes et Clostridium perfringens à la surface et en profondeur de la carcasse (Delhalleet al., 2010). Sanchez (2011) a obtenu sur des carcasses blanc bleu belge de type culard dont les animaux ont reçu une stimulation électrique, $20^{\circ} \mathrm{C}$ et $7^{\circ} \mathrm{C}$ en profondeur de la carcasse respectivement au bout de $16 \pm 1,4 \mathrm{~h}$ et $41,8 \pm 2,8 \mathrm{~h}$. Une stimulation électrique éventuelle ne serait donc pas utile pour descendre convenablement la température au sein des carcasses des races bovines $\mathrm{du}$ Bénin.

Le pH ultime dans la présente étude a été de $5,72 \pm 0,18$ et a été obtenu dans la majorité des cas après 24 heures post mortem. A $8 \mathrm{~h}$ de temps après abattage, il a été enregistré un $\mathrm{pH}$ de $5,97 \pm 0.19$. II est recommandé pour une bonne qualité de la viande un $\mathrm{pH}$ ultime de 5,5-5,7 à $24 \mathrm{~h}$ post-mortem et surtout que le $\mathrm{pH}$ soit descendu sous la valeur de 6,0-6,2 à $10 \mathrm{~h}$ de temps après abattage (Cartier et al., 2007). Sanchez (2011) a obtenu un pH ultime de $5,51 \pm 0,01$ à $8 \mathrm{~h}$ post mortem sur des carcasses ayant été stimulées électriquement. Malgré l'absence d'un étourdissement dans le procédé d'abattage des bovins à l'abattoir de Cotonou-PortoNovo, les conditions de pré-abattage en général n'ont pas engendré un épuisement des réserves en glycogène musculaire des animaux. II est possible que le régime alimentaire purement à base de fourrage ait apporté l'énergie suffisante pour avoir une évolution normale du $\mathrm{pH}$ post mortem. La température moyenne de la chambre froide $\left(3,29 \pm 0,5^{\circ} \mathrm{C}\right)$ et l'humidité relative moyenne $(76,22 \% \pm 3,81)$ sont dans les normes recommandées $(0$ à $4^{\circ} \mathrm{C}$ et 80 à $90 \%$ pour l'humidité relative) dans la littérature, pour un bon refroidissement (Rosset \& Roussel-Ciquard, 1984 ; James \& James, 2000). Toutefois, des variations allant jusqu'à $88,65 \%$ pour l'humidité relative et $6,72^{\circ} \mathrm{C}$ pour la température ont été observées à des moments précis de la journée correspondants aux heures d'ouverture de l'entreprise.

Effet de la race sur les qualités technologiques de la viande: La couleur de la viande est la première caractéristique qualitative de la viande perçue à l'achat. Le consommateur la considère comme un critère de fraicheur du produit (Clinquart et al., 2000 ; Coibion,
2008). Dans la présente étude, la viande de bovin de race Borgou a présenté dans l'ensemble des valeurs de paramètres de couleur $\left(L^{*}, a^{*} e t b^{*}\right)$ les plus élevées. Les trois races étant élevées sur pâturage naturel et sur des parcours différents, la différence de couleurs entre ces races peut être liée soit à la composition floristique du parcours ou soit à l'origine génétique des animaux (Insani et al., 2008 ; Mohamed et al., 2008 ; Schor et al., 2008). Chez les taurillons élevés sur pâturage naturel en région semi tropicale de l'Argentine, l'effet race a été observé entre les Criollo Argentino et les Braford sur chacun des paramètres de la couleur $\left(L^{*}, a^{*}\right.$ et $\left.b^{*}\right)$. De même, Dufey et Chambaz (2004) ont mis en évidence l'effet de la race sur la couleur de la viande, notamment, sur la luminosité, l'indice du rouge et l'indice du jaune $(P<0,05)$ chez 6 races pures (Angus, Simmental, Charolais, limousin, Blonde d'Aquitaine et Piémontais) engraissées en stabulation libre. Contrairement aux résultats de notre étude, l'effet race n'a pas été observé sur les paramètres de la couleur de la viande des taurillons Simmental, Bonsmara et Nguni élevés sur pâturage naturel dans les régions arides de l'Afrique du Sud (du Plessis \& Hoffman, 2007). La couleur de la viande peut être influencée par le poids de la carcasse à l'abattage : une carcasse plus lourde est associée à une viande plus rouge et plus sombre (Vestergaard et al., 2000). Dans la présente étude, les Zébu ont une carcasse plus lourde que les Borgou et les Lagunaire ont la carcasse, la plus légère. Cependant, ce sont les Borgou qui ont présenté les paramètres de couleur les plus élevés. En considérant l'origine génétique de la race Borgou qui est issue d'un croisement lointain entre les taurins et les Zébu (Youssao et al., 2009), on s'attendait également à ce que la race Borgou présente des valeurs intermédiaires entre celles des deux autres races. Pour l'instant, aucune hypothèse ne justifie la supériorité des valeurs des paramètres de couleur de la race Borgou.

La viande des bovins Lagunaire a une capacité de rétention d'eau plus élevée que celles des Borgou et des Zébus Peulh. La capacité de rétention de l'eau influence la jutosité de la viande (Hocquette et al., 2005). Dans la présente étude, la capacité de rétention d'eau est élevée chez la race Lagunaire avec une jutosité relativement élevée, bien que cette note ne soit pas significativement différente de celles des deux autres races. Les conditions d'abattage et post-abattage étant identiques dans la présente étude, les différences de capacité de rétention d'eau peuvent être liées au type génétique ou aux 

Lagunaire et Zebu sur pâturages naturels

caractéristiques musculaires. Les valeurs enregistrées ( 41,73 à 46,43\%) dans la présente étude sont inférieures à celles obtenues (80 à $81,1 \%$ ) par Chatibi (2011) sur la viande de bovins de race locale marocaine et Holstein et sur des races belges $(63,9 \%)$ obtenue par Delhalle et al. (2010) et inférieures à celles $(32,90 \% \pm 1,26)$ rapportées par Sanchez (2011). Des variations inter-races sont rapportées dans la littérature (Dufey \& Chambaz, 2004, du Plessis \& Hoffman, 2007 ;Orellana et al., 2009). Chez les taurillons élevés sur pâturage naturel en région semi tropicale de l'Argentine, l'effet race a été observé entre les Criollo Argentino et les Braford sur la perte de jus à la cuisson (Orellana et al., 2009). En revanche, aucune différence n'a été observée entre les pertes de jus à l'écoulement et la capacité de rétention de l'eau des deux races (Orellana et al., 2009). L'effet race n'a pas été observé sur la perte de jus au ressuyage, la perte de jus à la cuisson entre les taurillons Simmental, Bonsmara et Nguni élevés sur pâturage naturel des régions arides de l'Afrique du Sud (du Plessis \& Hoffman, 2007). Engraissées en stabulation libre, les Simmental et surtout les Angus ont les pertes de jus à l'écoulement les plus faibles, ce qui reflète un meilleur pouvoir de rétention d'eau. Les pertes les plus élevées se situent chez les Blonde d'Aquitaine et chez les Piémontais, lorsque ces derniers sont abattus avec la note 3 d'état d'engraissement (Dufey \& Chambaz, 2004). Enfin, Oliván et al. (2001) ont également observé une relation entre le niveau d'engraissement et la capacité de rétention de l'eau. Bien que les différences ne soient pas significatives, les forces de cisaillement les plus élevées ont été obtenues chez les Borgou et les plus faibles ont été enregistrées chez les Lagunaire. Cette différence est en accord avec les résultats obtenus sur chacune des trois races par Salifou et al. (2013). La différence de texture entre les trois races est due à leur teneur en collagène. Selon Salifou et al. (2013), la teneur en collagène total des Borgou (5,2 $\mathrm{\mu g} \mathrm{OH}$-proline/mg MS de muscle) a été plus élevée que celles des Zébu Peulh $(3,1$ $\mu \mathrm{g} \mathrm{OH}$-proline/mg MS de muscle) et des Lagunaires (3,2 $\mu \mathrm{g} \mathrm{OH}$-proline/mg MS de muscle). Dans la littérature, de nombreux auteurs ont rapporté l'effet race sur la tendreté de la viande bovine. Chez les taurillons élevés sur pâturage naturel en région semi tropicale de l'Argentine, l'effet race a été observé entre les Criollo Argentino et les Brasford sur la tendreté (Orellana et al., 2009). L'effet race n'a pas été observé sur la tendreté et la force de cisaillement entre les taurillons Simmental, Bonsmara et
Nguni élevés sur pâturage naturel des régions arides de l'Afrique du Sud (du Plessis \& Hoffman, 2007). Les races spécialisées dans la production de la viande (Limousin et blonde d'Aquitaine) ont une faible valeur de force de cisaillement par rapport aux autres races (Holstein et Old Brown Swis) (Monsón et al., 2004). La viande de Santa Gertrudis est moins tendre que celles de la race Sanga, Afrikaner, Nguni, Brown Swiss, Pinzgauer et Bonsmara (Strydom et al., 2000). La meilleure note de tendreté de la viande des Lagunaires par rapport à celle des deux autres races confirme les résultats obtenus dans la présente étude sur la force maximale de cisaillement. Malgré l'absence de différence significative observée entre la note de jutosité de chacune des trois races, celle des Lagunaires est plus élevée et confirme la perte de jus à la cuisson plus importante obtenue.

Variation intra-race des qualités technologiques au cours de la maturation: Pour chacune des trois races, la luminance et l'indice du jaune ont eu la même tendance de variation (augmentation du jour d'abattage au jour 2 post-mortem et diminution du jour 2 au 8 e jour postmortem). Cette tendance amène à penser que l'évolution de la luminance et de lindice du jaune au cours de la maturation n'est pas fonction de la race mais beaucoup plus des conditions de conservation. Vautier et al. (2004) ont obtenus la même tendance de l'indice du jaune sur la viande de porcs Label Rouge et Standard. Du jour d'abattage au jour 2 post mortem, la teneur de l'indice du rouge a augmenté de manière significative chez les lagunaires et de manière non significative chez les Borgou et les Zébu. Au jour 8 post mortem, elle a chuté significativement chez les trois races. Ces résultats indiquent que chez les bovins étudiés, la variation de l'indice a"du muscle Longissimus thoracis est fonction du temps de maturation. II augmente légèrement au bout des premières 48 heures et diminue au bout de 8 jours post-mortem. II serait intéressant de confirmer ou d'infirmer cette observation en élargissant l'étude à d'autres races avec une taille plus importante de l'échantillon et aussi de déterminer avec précision à quel jour précis de la maturation lindice du rouge connait une diminution.

L'évolution de l'angle de la teinte et de la saturation pour chacune des trois races dans la présente étude indique que, une oxydation de la viande marquée par une diminution de l'indice du rouge et de la chromaticité ne s'accompagne pas forcément d'une augmentation de la teinte tel que stipulé par Rennere (2006). Cuvelier et al. 

Lagunaire et Zebu sur pâturages naturels

(2006) a obtenu chez les bovins Blanc Bleu Belge et Aberdeen Angus, un effet de la race sur la couleur de la viande du jour 2 au jour 8 après l'abattage et cette tendance a été observée dans la présente étude lors de la maturation. $\mathrm{A} 1$ heure post mortem, le pH le plus élevé a été obtenu chez les Zébu. A 48 heures et à 8 jours postmortem, les $\mathrm{pH}$ les plus faibles ont été enregistrés chez la même race. On note ainsi une diminution rapide du pH s'expliquant peut-être par le fait que les Zébu sont

\section{CONCLUSION}

Les caractéristiques technologiques et organoleptiques de la viande des bovins de races Lagunaire, Borgou et Zébu Peulh présentent une grande variabilité. Ainsi, les Zébu Peulh ont une température de refroidissement supérieure aux Borgou et les Lagunaire ont les températures les plus faibles tout au long de la maturation. L'écart entre la température en profondeur et celle en surface de la carcasse diminue chez chacune des trois races au cours de la maturation en chambre froide. La viande de bovin de race Borgou est plus claire que celle des Lagunaire et des Zébu Peulh pendant que l'indice du rouge varient d'une race à l'autre au cours de la maturation. Les Zébu Peulh présentent les plus faibles indice du jaune alors que les Borgou ont les indices de jaune les plus élevés quel que soit le jour de mesure. La teinte de la viande est identique chez les trois races le jour de l'abattage. Le pH de la viande des trois races est

\section{REMERCIEMENTS}

Les auteurs remercient la Coopération Universitaire au Développement (CUD) pour son appui à la réalisation de ces travaux à travers le troisième Programme Quinquennal (P3) de la Coopération Universitaire

\section{RÉFÉRENCES BIBLIOGRAPHIQUES}

Cartier P. and Moevil, 2007.La qualité des carcasses et des viandes de gros bovins. Compte rendu final $n^{\circ} 170532$ 022, Département Techniques d'Elevage et Qualité, Service Qualité des Viandes, France. 2007, ISSN: 1773-4738, 70p. www.agrireseau.qc.ca/.../qualite_carcasse_vian de_bovin_2008

Chatibi S, 2011. La filière viande bovine au Maroc: Quelle place pour l'élevage traditionnel et quelles bases de qualification pour la viande locale? Thèse de doctorat, Université de Corse, 230pp. les animaux les plus lourds des trois races. Selon Dufey \& Chambaz (2004), les races à fort développement musculaire comme les Piémontais se distinguent des races Blonde d'Aquitaine par une chute plus rapide de la température dans le muscle Longissimus thoracis entre une et trois heures post mortem (Dufey \& Chambaz, 2004). Cette évolution est semblable pour les Angus et les Simmental (Dufey \& Chambaz, 2004).

identique pour les mêmes jours de mesure. La viande des bovins Lagunaires perd plus d'eau à l'égouttage et à la cuisson que celles des Zébu Peulh et des Borgou. Le mode de cuisson n'influence pas la tendreté, la jutosité, la flaveur et la note globale d'acception par le consommateur. La flaveur et la jutosité de la viande de chacune des trois races ne varient pas d'une race à l'autre. Par contre, la viande des Lagunaire est plus tendre que celle des Borgou et des Zébu. En prenant en compte la flaveur, la jutosité, la tendreté et la note d'acceptation de la viande de la Lagunaire par le consommateur, la viande de bovin Lagunaire a une qualité technologique, organoleptique et sensorielle meilleure à celles des Borgou et des Zébu Peulh. Une étude sur l'amélioration de la qualité de la viande des bovins Borgou et des Zébu Peulh est nécessaire pour rendre la filière bovine plus compétitive.

Institutionnelle (CUI) du Conseil Interuniversitaire de la Communauté Française de Belgique (CIUF). Les remerciements sont également adressés aux responsables de l'Activité UAC01.

Clinquart A, Leroy B, Dottreppe O, Hornick JL, Dufrasne IL, Istasse L, 2000. Les facteurs de production qui influencent la qualité de la viande des bovins Blanc Bleu belge. L'élevage du Blanc Bleu Belge, CESAM, 19pp.

Coibion L, 2008. Acquisition des qualités organoleptiques de la viande bovine: adaptation à la demande du consommateur. Mémoire pour l'obtention du grade de Docteur Vétérinaire. Ecole Nationale Vétérinaire de Toulouse, 97pp.

Collignon B, 2009. Étude du refroidissement des carcasses de gros bovins de race Blanc Bleu 
Belge: conditions de refroidissement et courbes temps-température de référence. Mémoire présenté en vue de l'obtention du diplôme de Master complémentaire en médecine vétérinaire spécialisée. Université de Liège, 141pp.

CountryStat/Benin, 2012. Base de données statistique, consulté à l'adresse, http://countrystat.org/ben ou http://www.fao.org/economic/ess/countrystat/en/

CSIRO, 1989. Australian Division of Food Processing Meat Research Laboratory (1989). Bone taint. Meat Research News Letter5: 1-2.

Cuvelier C, Cabaraux JF, Dufrasne I, Clinquart A, Hocquette JF, Istasse L, Hornick JL, 2006. Performance, slaughter characteristics and meat quality of young bulls from Belgian Blue, Limousin and Aberdeen Angus breeds fattened with a sugar-beet pulp or a cereal-based diet. Animal Science 82: 125-132.

D.E, 2011. Direction de l'Elevage, Rapport annuel d'activités, Bénin, 109pp

Delhalle L, Collignon B, Dehard S, Imazaki P, Daube $G$,Clinquart A, 2010. Chilling of carcasses from double muscled cattle: time-temperature evolution and predictive modelling of growth of listeria monocytogenes and clostridium perfringens. International Congress of Meat science and Technology (Jeju), Corea, August, 15-20, 2010: 4pp.

Doko AS, Farougou S, Salifou S, Ehilé E, Geerts S, 2010. Dynamique des infections trypanosomiennes chez des bovins Borgou à la ferme de l'Okpara au Bénin. Tropicultura 28 (1): $37-43$.

DuPlessis I. and Hoffman LC, 2007. Effect of slaughter age and breed on the carcass traits and meat quality of beef steers finished on natural pastures in the arid subtropics of South Africa. South African Journal of Animal Science 37 (3): 143-153.

Dufey PA. and Chambaz A, 2004. Qualité de la viande de bœufs de six races à viande. Agrocopeliebefield-Posieux, Revue suisse agricole 36 (6) : 265-274.

Farougou S, Tassou AW, Tchabodé DM, Kpodékon M. Boko C, Youssao AKI, 2007. Tiques et hémoparasites du bétail dans le nord-Bénin. Revue Médecine Vétérinaire 158 (8-9): 463-467.
Farougou S, Adakal H, Biguezoton AS, Boko C, 2012. Prévalence de l'infection d'Amblyomma variegatum par Ehrlichia ruminantium dans les élevages extensifs du Bénin.Revue Médecine Vétérinaire163 (5) : 261-266.

Farougou S, Agbadjè P, Kpodekon M, Adoligbe C, Akakpo AJ, 2006. Prévalence de la tuberculose bovine dans les fermes d'Etat de Samiondji et de Bètècoucou au Bénin. Revue Africaine Santé et Production Animales $4:$ 27-31.

Hocquette JF, Cassar-Malek I, Listrat A, Jurie C, Jailler $R$, Picard $B, 2005$. Evolution des recherches sur le muscle des bovins et la qualité sensorielle de leur viande. II. Influence des facteurs d'élevage sur les caractéristiques musculaires. Cahier d'Agriculture14: 365-372.

Honikel KO, 1987. How to measure the water-holding capacity of meat? Recommendation of standardized methods. In: V.G. Tarrant (Editor). Evaluation and control of meat quality in pigs.Martinus Nijhoff Pub, Den Haag: 129-142.

Insani EM, Eyherabide A, Grigioni G, Sancho AM, Pensel NA, Descalzo AM, 2008. Oxidative stability and its relationship with natural antioxidants during refrigerated retail display of beef produced in Argentina. Meat Science79: 444-452.

James SJ.and James C, 2000. Meat refrigeration. In: CRC Press LLC, Boca Raton, USA, 524 pp.

Koutinhouin B, Youssao AKI, Houehou AE, Agbadje PM, 2003.Prévalence de la brucellose bovine dans les élevages traditionnels encadrés par le Projet pour le Développement de l'Elevage (PDE) au Bénin. Revue Médecine Vétérinaire154 (4): 271276.

Koutinhouin GB, Youassao AKI, Tobada P, Kpodekon TM, Adimatin V, 2009. Influence de l'indice de température et d'humidité relative de l'air sur la fécondité de la vache Borgou élevée selon deux modes d'élevage au Bénin. International Journal of Biology and Chemical Science 3(6): 13361345.

Koutinhouin GB, Youssao AKI, KpodekonTM, Gantoli YG, 2010. Influence de la traite précoce des vaches sur la croissance pondérale et l'état sanitaire des veaux en élevage traditionnel : cas de la zone périurbaine de Natitingou (Bénin). Livestock Research for Rural Development, 22 $\underline{(02)}$ 
2010.http://www.llrd.org/l/rrd22/2/kout22024.htm

Mohamed A, Jamilah B, Abbas KA, Abdul Rahman R, 2008. A review on some factors affecting colour of fresh beef cuts. Journal of Food and Agricultural Environment6: 181-186.

Monsón F, Sañudo C, Sierra I, 2004.Influence of cattle breed and ageing time on textural meat quality. Meat Science68(4): 595-602.

Olivan M, Martinez A, Garcia P, Noval G, Osoro K, 2001.Estimation of the carcass composition of yearling bulls of Asturiana de los Valles breed from the dissection of a rib joint. Meat Science 57: 185-190.

Orellana C, Peña F, García A, Perea J, Martos J, Domenech V, Acero R,2009. Carcass characteristics, fatty acid composition, and meat quality of Criollo Argentino and Braford steers raised on forage in a semi-tropical region of Argentina. Meat Science 81: 57-64

Oury MP, Picard B, Istasse L, Micol D, Dumont R, 2007.Mode de conduite en élevage et tendreté de la viande bovine. INRA Production Animale 20 : 309-326.

Règlement (CE) $n^{\circ}$ 853/2004 du Parlement européen et du Conseil du 29 avril 2004 fixant des règles spécifiques d'hygiène applicables aux denrées alimentaires d'origine animale. Journal officiel $n^{\circ} L 139$ du 30/04/2004 p. 0055 - 0205. http://eur-

lex.europa.eu/LexUriServ/LexUriServ.do?uri=CE LEX:32004R0853:FR:HTML

Renerre M, 2006. La mesure de la couleur de la viande. Journées Science du Muscle et Technologie de la Viande 257pp.

Rosset R, Roussel-Ciquard N, 1984. Le froid dans la filière viande. In Rosset, R., Les viandes: Hygiène - Technologie. Paris: Informations techniques des services techniques vétérinaires : 217-224.

Salifou CFA, Dahouda M, Ahounou GS, Kassa SK, Tougan PU, Farougou S, Mensah GA, Salifou S, Clinquart A, Youssao AKI, 2012a. Evaluation of carcass traits of Lagunaire, Borgou and Zebu Fulani bulls raised on natural pasture in Benin. The Journal of Animal and Plant Sciences, 22 (4): 857-871.

Salifou CFA, Dahouda M, Ahounou GS, Kassa SK, Tougan PU, Farougou S, Mensah GA, Salifou S,
Clinquart A, Youssao AKI, 2012b. Assessment of offal components of Lagunaire, Borgou and Zebu Fulani bulls raised on natural pasture and analysis of macroscopic lesions associated with potential hazards for the consumer. African Journal of Agricultural Research (soumis).

Salifou CFA, Dahouda M, Ahounou GS, Kassa SK, Tougan PU, Farougou S, Mensah GA, Salifou S, Clinquart A, Youssao AKI. 2012c. Relationships between carcass characteristics and offal components of Lagunaire, Borgou and Zebu Fulani bulls raised on natural pasture. International Journal of Biosciences2 (11), 117128.

Sanchez MM, 2011. Influence of two different timetemperature. Evolution during carcasse chilling and two packaging techniques on meat quality in double muscled Belgian Blue cattle. Mémoire présenté en vue de l'obtention du grade de formation doctorale en sciences vétérinaires: 38 pp.

SAS, 2006.SAS/STAT User's guide, vers, 6, 4thed, Cary, NC, USA, SAS Inst.

Schor A, Cossu ME, Picallo A, Martinez Ferrer J, GrigeraNaon JJ, Colombatto D, 2008. Nutritional and eating quality of Argentinean beef. Meat Science 79: 408-422.

Strydom PE, Naede RT, Smith MF, Scholtz MM, van Wyk $J B, 2000$. Characterization of indigenous African cattle breeds in relation to meat quality traits. Meat Science 55: 79-88.

Vautier A, Minvielle B, Boulard J, Bouyssiere M, Houix Y, 2004. Viandes déstructurées: Effets du système d'abattage et des conditions météorologiques. Techni.Porc 27 (3): 19-23.

Vestergaard $M$, Therkildsen $M$, Henckel $P$, Jensen $L R$, Andersen HR, Sejrsen K, 2000. Influence of feeding intensity, grazing and finishing feeding on meat and eating quality of young bulls and the relationship between muscle fibre characteristics, fibre fragmentation and meat tenderness. Meat Science 54:187-195.

Youssao AKI, Koutinhouin GB, Kpodekon TM, Agnandjo H, Toure Z, Ahissou A,Renand G, 2007. Variabilité génétique des performances de croissance et des mesures corporelles de jeunes bovins de race Borgou à la Ferme 
Salifou et al.... J. Appl. Biosci. 2013. Evaluation de la qualité de la viande de bovins des races Borgou, Lagunaire et Zebu sur pâturages naturels

d'Elevage de l'Okpara. Revue Africaine Santé et Production Animales 5, (3-4): 157-165.

Youssao AKI, Koutinhouin GB, KpodekonTM, Agnandjo $H$, Toure Z, Ahissou A, 2009. Influence d'une sélection phénotypique sur les performances de croissance et les caractères de développements musculaire et squelettique de jeunes bovins de race Borgou à la Ferme d'Elevage de l'Okpara
(Bénin). Annales de MédecineVétérinaire153: 105-111.

Zoffoun GA, Salifou $S$, Houinato $M$, Sinsin $A B$, 2011b.Interactions ticks, hosts and pastures: Case of the Girolando dairy cattle and the artificial pastures of Panicum maximum and Panicum maximum var. C1. Journal of Agricultural and Science Technology 5 (4): 433442. 